International Journal of Pure and Applied Mathematics

Volume 89 No. 2 2013, 147-151

ISSN: 1311-8080 (printed version); ISSN: 1314-3395 (on-line version)

url: http://www.ijpam.eu

doi: http://dx.doi.org/10.12732/ijpam.v89i2.3

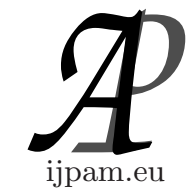

\title{
SOME REMARKS ON $n$-POWER CLASS(Q) OPERATORS
}

\author{
Krutan Rasimi ${ }^{1 \S}$, Luigj Gjoka ${ }^{2}$ \\ ${ }^{1}$ Faculty of Natural Sciences and Mathematics \\ State University of Tetova \\ Bul. Ilinden nn, 1200 Tetovo, MACEDONIA \\ ${ }^{2}$ Faculty of Mathematical Engineering and Physical Engineering \\ Polytechnic University of Tirana \\ Bul. "Dëshmorët e Kombit" Square "Mother Teresa" 4, 1001 Tirana,
} ALBANIA

\begin{abstract}
The purpose of the present paper is to give some results relate to a class of linear bounded operators, known as $n$-power class $(\mathrm{Q})$ operators acting on infinite complex separable Hilbert space $H$. $n$-power class(Q) operators is extension of class $(\mathrm{Q})$ operators and class of $n$-normal operators The class of $n$-power class (Q) operators was defined by S. Panayappan and N. Sivamani in [1], where they have given some of their properties. Based, mainly, on [1], [3], [4], we contribute with some others properties of such operators.
\end{abstract}

AMS Subject Classification: 47B20

Key Words: Hilbert space, quasi $n$-normal operators, $n$-power class(Q) operators, doubly commutative

\section{Introduction}

In this paper we give some results about $n$-power class $(\mathrm{Q})$ operators defined on [1], where authors also have depicted the elementary properties of such operators. Firstly, let give some introductory concepts. Throughout this text we denote with $H$ a complex infinite separable Hilbert space and with $L(H)$ we denote the algebra of all bounded linear operators acting on $H$. We say that an

Received: June 12, 2013

(c) 2013 Academic Publications, Ltd.

$\S_{\text {Correspondence author }}$ url: www.acadpubl.eu 
operator $T$ from $L(H)$ is a normal operator if is commutative with his adjoint operator $T^{*}$, that is, if $T^{*} T=T T^{*}$ For operator $T$ from $\left.L(H)\right)$ we say that is $n$-normal operator if $T^{n} T^{*}=T^{*} T^{n}$ holds. It is evident that for $n=1$ we obtain the class of normal operators. We say that operator $T$ is $n$-power quasi normal if $T^{n}\left(T^{*} T\right)=\left(T^{*} T\right) T^{n}$. Operator $T$ is quasi $n$-normal if $T\left(T^{*} T^{n}\right)=\left(T^{*} T^{n}\right) T$. Finally, we say operator $T$ is of class(Q) if $T^{* 2} T^{2}=\left(T^{*} T\right)^{2}$, and $T$ is $n$-power class(Q) operator if $T^{* 2} T^{2 n}=\left(T^{*} T^{n}\right)^{2}$.

\section{Main Results}

In [1], Theorem 2.5, authors have proved that if $T$ from $L(H)$ is $n$-normal operator, then it is also $n$-power class $(\mathrm{Q})$ operator. By example they proved that the converse is not true. At this point, we claim that with additional condition of invertibility on $n$-power class $(\mathrm{Q})$ operator $T$, he must be $n$-normal operator. That is, we say

Theorem 2.1. Let $T$ be bounded linear operator. If $T$ is $n$-power $\operatorname{class}(Q)$ operator and has inverse than $T$ is $n$-normal operator.

Proof. Let $T \in L(H)$ and let $T$ be a $n$ power class(Q) operator, that is, $T^{* 2} T^{2 n}=\left(T^{*} T^{n}\right)^{2}$. We can write that $T^{* 2} T^{2 n}=T^{*} T^{n} T^{*} T^{n}$. Now, since $T$ has inverse it is elementary that the adjoint operator $T^{*}$ also have inverse and he is $\left(T^{*}\right)^{-1}=\left(T^{-1}\right)^{*}$. If we multiply $T^{* 2} T^{2 n}=T^{*} T^{n} T^{*} T^{n}$ with $\left(T^{*}\right)^{-1}$ from the left we obtain $T^{*} T^{2 n}=T^{n} T^{*} T^{n}$. This relation is equivalent to $T^{*} T^{n} T^{n}=T^{n} T^{*} T^{n}$. Further, we multiply $n$-time with $T^{-1}$ from the right, or, equivalently, we multiply with $\left(T^{n}\right)^{-1}$ from the right and this operation will results to $T^{*} T^{n}=T^{n} T^{*}$. That is, with the above condition $n$-power class $(\mathrm{Q})$ operator is $n$-normal operator. The proof is completed.

In [1] authors have proved that $n$-power class(Q) operator and $(n+1)$ power class $(\mathrm{Q})$ operator are not comparable. Specifically, they support this by matrices in Example 2.7 and Example 2.8. Further, they proved in Theorem 2.9 ([1]), that if $T$ is $n$-power class(Q) operator and $T$ is quasi $n$-normal operator, then $T$ is $(n+1)$-power class $(\mathrm{Q})$ operator. Also, by Example 2.10 they proved that the condition that $T$ is quasi $n$-normal operator is necessery.

We generalize this result and claim that:

Theorem 2.2. Let $T$ be bonded linear operator on $H$. If $T$ is $(n+k)$ power class $(Q)$ operator and in same time is quasi $n$-normal operator, then $T$ is $(n+k+1)$-power class $(Q)$ operator for any $k \geq 0$ 
Proof. Let $T$ be a bonded linear operator on $H$. Suppose that $T$ is $(n+$ $k)$-power class $(\mathrm{Q})$ operator and quasi $n$-normal operator. For given conditions we have that operator $T$ satisfy the following $T^{* 2} T^{2(n+k)}=\left(T^{*} T^{n+k}\right)^{2}$ and $T\left(T^{*} T^{n}\right)=\left(T^{*} T^{n}\right) T$. We need to prove that $T^{* 2} T^{2(n+k+1)}=\left(T^{*} T^{n+k+1}\right)^{2}$.

Let evaluate the left hand site of last relation, we have

$$
\begin{aligned}
T^{* 2} T^{2(n+k+1)} & =T^{* 2} T^{2(n+k)} T^{2} \\
& =\left(T^{*} T^{(n+k)}\right)^{2} T^{2} \\
& =T^{*} T^{(n+k)} T^{*} T^{(n+k)} T T \\
& =T^{*} T^{n} T^{k} T^{*} T^{n} T^{k} T T \\
& =T^{*} T^{n} T^{k} T^{*} T^{n} T T^{k} T \\
& =T^{*} T^{n} T^{k} T T^{*} T^{n} T^{k} T \\
& =T^{*} T^{n+k+1} T^{*} T^{n+k+1} \\
& =\left(T^{*} T^{n+k+1}\right)^{2} .
\end{aligned}
$$

For $k=0$ we have Theorem 2.9 in [1].

In Theorem 2.11, in [1] authors have proved that direct sum and product of $m n$-normal operators are $n$-power class $(\mathrm{Q})$ operators. It is well known that product of two operators of same class is not necessary operator from the same class. Even in the case when operators commute to each other is not good enough to ensure this. For example see [3]. In other hand, if we suppose that operator $T$ and operator $S$ are doubly commutative, that is $T S=S T$ and $T S^{*}=S^{*} T$, then we can claim that

Theorem 2.3. Let $T, S$ are bounded linear operators from $L(H)$. If $T$ and $S$ are such n-power class $(Q)$ operator that doubly commutes, that is, $T S=S T$ and $T S^{*}=S^{*} T$, then operator $T S$ is $n$-power class $(Q)$ operator.

Proof. Let $T$ and $S$ be $n$-power class(Q) operator. Let $T$ doubly commutes with $S$. In order to prove that $(T S)^{* 2}(T S)^{2 n}=\left[(T S)^{*}(T S)^{n}\right]^{2}$ we begin with left hand site as follow $(T S)^{* 2}(T S)^{2 n}=S^{*} T^{*} T^{*} S^{*} \underbrace{T S T S \ldots T S}_{2 n-t i m e}$. Since $T$ doubly commutes with $S$ we can write that $(T S)^{* 2}(T S)^{2 n}=S^{*} S^{*} T^{*} T^{*} \underbrace{T S T S \ldots T S}_{2 n-t i m e}$. Now, from the same reason, that is, $T S=S T$ and $T S^{*}=S^{*} T\left(\right.$ or $\left.T^{*} S=S T^{*}\right)$, by successive remove on the left of each of $S$, we obtain

$$
(T S)^{* 2}(T S)^{2 n}=S^{*} S^{*} \underbrace{S S \ldots S}_{2 n-\text { time }} T^{*} T^{*} \underbrace{T T \ldots T}_{2 n \text {-time }}
$$




$$
\begin{aligned}
& =S^{* 2} S^{2 n} T^{* 2} T^{2 n}(\text { since } T \text { and } S \text { are n-power class(Q) operator) } \\
& =\left(S^{*} S^{n}\right)^{2}\left(T^{*} T^{n}\right)^{2} \\
& =S^{*} S^{n} S^{*} S^{n} T^{*} T^{n} T^{*} T^{n} \text { (since } T \text { and } S \text { doubly commutes) } \\
& =S^{*} T^{*} S^{n} T^{n} S^{*} T^{*} S^{n} T^{n} \\
& =(T S)^{*}(T S)^{n}(T S)^{*}(T S)^{n} \\
& =\left[(T S)^{*}(T S)^{n}\right]^{2}
\end{aligned}
$$

The proof is completed.

Remarks 2.4. Also, under the same conditions, operator $S T$ is $n$-power class $(\mathrm{Q})$ operator.

Corollary 2.5. If $T$ is $n$-power class $(Q)$ operator such that $T$ doubly commute with an isometric operator $S$, then $T S$ is $n$-power class $(Q)$ operator.

Proof. The proof of this statement is similar to that of Theorem 3. From given conditions we have

$$
\begin{aligned}
& (T S)^{* 2}(T S)^{2 n}=S^{*} T^{*} S^{*} T^{*} \underbrace{T S T S . . . T S}_{2 n-\text { time }} \\
& =S^{*} S^{*} S S T^{* 2} T^{2 n} \underbrace{S S \ldots S}_{2 n-2-t i m e} \text { (since } T \text { and } S \text { doubly commutes) } \\
& =T^{* 2} T^{2 n} S^{2 n-2} \text { (since } T \text { is } n \text {-power class(Q) operator) } \\
& =T^{*} T^{n} T^{*} T^{n} S^{2 n-2} \text { (since } S \text { is isometric operator and } \\
& T \text { and } S \text { doubly commutes) } \\
& =S^{*} S T^{*} S^{n-1} T^{n} S^{*} S T^{*} S^{n-1} T^{n} \text { (since } T \text { and } S \\
& =S^{*} T^{*} S^{n} T^{n} S^{*} T^{*} S^{n} T^{n} \\
& =(T S)^{*}(T S)^{n}(T S)^{*}(T S)^{n} \\
& =\left[(T S)^{*}(T S)^{n}\right]^{2} \text {. } \\
& \text { doubly commutes) }
\end{aligned}
$$

\section{References}

[1] S. Panayappan, N. Sivamani, On $n$ power class(Q) operators, Int. Journal of Math. Analysis, 6, No. 31 (2012), 1513-1518.

[2] S.A. Alzuraiqi, A.B. Patel, On n-normal operators, General Mathematics Notes, 1, No. 2 (2010), 61-73. 
[3] C.S. Kubrusly, Hilbert Space Operators, A problem Solving Approach, Birkhauser, Boston (2003).

[4] T. Veluchamy, A. Devika, Some properties of quasi-*paranormal operators, Journal of Modern Mathematics and Statistics, 1, No-s: 1-4 (2007), 35-38. 
